CHAPTER 15

\title{
The Taliban
}

Jon Armajani

Due to rights restrictions,

this chapter is not available

in the digital edition of the book. 